\title{
Digital Distances and Integer Sequences
}

\author{
Nicolas Normand $^{1}$, Robin Strand ${ }^{2}$, and Pierre Evenou ${ }^{1}$ \\ ${ }^{1}$ LUNAM Université, Université de Nantes, IRCCyN UMR CNRS 6597, \\ Polytech Nantes, Rue Christian Pauc, La Chantrerie, \\ 44306 Nantes Cedex 3, France \\ 2 Centre for Image Analysis, Uppsala University, Sweden
}

\begin{abstract}
In recent years, the theory behind distance functions defined by neighbourhood sequences has been developed in the digital geometry community. A neighbourhood sequence is a sequence of integers, where each element defines a neighbourhood. In this paper, we establish the equivalence between the representation of convex digital disks as an intersection of half-planes ( $\mathcal{H}$-representation) and the expression of the distance as a maximum of non-decreasing functions.

Both forms can be deduced one from the other by taking advantage of the Lambek-Moser inverse of integer sequences.

Examples with finite sequences, cumulative sequences of periodic sequences and (almost) Beatty sequences are given. In each case, closedform expressions are given for the distance function and $\mathcal{H}$-representation of disks. The results can be used to compute the pair-wise distance between points in constant time and to find optimal parameters for neighbourhood sequences.
\end{abstract}

\section{Introduction}

A discrete distance function is often defined by using the concept of minimal cost paths obtained by weighted paths and/or neighbourhood sequences [10. The paths in the traditionally used city-block $d_{4}$ and chessboard $d_{8}$ distance functions [9] are restricted to the four (and eight) closest neighbours of each grid point in $\mathbb{Z}^{2}$. In $5[2$, different weights for closest neighbours and diagonal neighbours are considered giving the weighted distances and in 1022 , the neighbourhood that is allowed in each step is not constant, but defined by a neighbourhood sequence. In [11, both weights and neighbourhood sequences are used to define the distance function. One benefit with the path-generated, discrete, distance functions over the Euclidean distance is when computing point-to-point distances on non-convex domains with the constrained distance transform. The cDT labels each object pixels with the distance to the closest seed pixels, where paths that define the distances are not allowed to cross obstacle pixels. With minimal cost-path distances, the cDT can be computed using standard shortestpath techniques for weighted graphs resulting in a linear time algorithm. A bucket sorting implementation of the Dijkstra's algorithm is used in [12]. See also 11 .

R. Gonzalez-Diaz, M.-J. Jimenez, B. Medrano (Eds.): DGCI 2013, LNCS 7749, pp. 169-179, 2013.

(C) Springer-Verlag Berlin Heidelberg 2013 
A neighbourhood sequence is an integer sequence, but the link between the theory on integer sequences and the theory on discrete distance functions has not been examined in detail yet. In this paper we will apply the theory on integer sequences and use the Lambek-Moser inverse, which is defined for non-decreasing integer sequences, to express the weighted ns-distance. We apply the so-obtained expression to parameter optimization. In [3], neighbourhood sequences on the form of Beatty sequences are considered.

By establishing a link between integer (neighbourhood) sequences and the Lambek-Moser inverse, this paper enables the use of Beatty sequences for distance computations. The inverse of a Beatty sequence can be written in closed form and any element can be computed in constant time. This property is useful for efficient computation (using, for example, wave-front propagation, constrained (geodesic) DT), 11. Moreover, we give $\mathcal{H}$-representation of digital disks, which is useful for, for example, parameter optimization by the link between digital disks and their enclosing polyhedron given in this paper.

\section{Integer Sequences - The Lambek-Moser Inverse and Beatty Sequences}

We denote sequences of integers as $f=(f(1), f(2), \ldots)$. The Lambek-Moser inverse of the non-decreasing sequence $f$, denoted by $f^{\dagger}$, is a non-decreasing sequence of integers defined by [4]:

$$
\forall m, n \in \mathbb{N}_{+}^{2}, f(m)<n \Leftrightarrow f^{\dagger}(n) \nless m \Leftrightarrow f^{\dagger}(n) \geq m .
$$

In Lambek and Moser's paper, $f$ and $f^{\dagger}$ are only defined for positive indices. However, (1) still holds without altering $f^{\dagger}$ if we extend the domain of $f$ to $\mathbb{Z}$ with $f(m)=0, \forall m \leq 0$ (the same applies to $f^{\dagger}$ ):

$$
\forall m \in \mathbb{Z}, \forall n \in \mathbb{N}_{+}, m \leq 0 \text { or } f(m)<n \Leftrightarrow f^{\dagger}(n) \geq m
$$

Proposition 1. $f^{\dagger}(f(m)+1)+1$ is the rank of the smallest term greater than $m$ where $f$ increases [7,8].

If we extend $f$ with $f(0)=0$, and define $g$ by $g(0)=0, g(n+1)=f^{\dagger}(f(g(n))+$ $1)+1$, then $f(g(n))$ takes, in increasing order, all the values of $f$, each one appearing once [7]8].

A Beatty sequence is the sequence obtained by taking the integer parts of the multiples of an irrational constant $\tau:(\lfloor\tau\rfloor,\lfloor 2 \tau\rfloor,\lfloor 3 \tau\rfloor, \ldots)$ [1]. Beatty sequences with parameter $\tau \geq 1$ are non-decreasing. We call Rational Beatty sequence the sequence produced with a rational parameter $\tau$. Hajdu introduced the use of Beatty sequences in the context of discrete distances [3].

The inverse of the Beatty sequence $f: m \mapsto\lfloor\tau m\rfloor$ with a scalar $\tau$, is $f^{\dagger}$ : $n \mapsto\left\lceil\frac{n}{\tau}-1\right\rceil$ whereas the inverse of $f: m \mapsto\left\lfloor\frac{a m}{b}+c\right\rfloor$ is $f^{\dagger}: m \mapsto\left\lfloor\frac{b m}{a}-c-1\right\rfloor$ where $a, b$ and $c$ are integers. 


\section{Distance Functions and $\mathcal{H}$-Representation of Balls}

\subsection{Discrete Distances}

Definition 1 (Discrete distance and metric). Consider a function $d: \mathbb{Z}^{n} \times$ $\mathbb{Z}^{n} \rightarrow \mathbb{N}$ and the following properties $\forall x, y, z \in \mathbb{Z}^{n}, \forall \lambda \in \mathbb{Z}$ :

1. positive definiteness $d(x, y) \geq 0$ and $d(x, y)=0 \Leftrightarrow x=y$,

2. symmetry $d(x, y)=d(y, x)$,

3. triangle inequality $d(x, z) \leq d(x, y)+d(y, z)$,

$d$ is called $a$ distance function, or distance, if it verifies conditions 1 and 2 and a metric with conditions 1 to 3 .

Definition 2 (Closed Ball). For a given distance function $d$, the closed ball $D$ with radius $r$ centered in $c$ is the following set of points of $\mathbb{Z}^{n}$ :

$$
D(c, r)=\{p: d(c, p) \leq r\} .
$$

A series of disks is increasing with respect to set inclusion:

$$
\forall r \in \mathbb{N}, D(c, r) \subseteq D(c, r+1) .
$$

Moreover, a discrete distance function is completely described by the sequence of its balls.

$$
d(O, p)=\min \{r: p \in D(O, r)\}
$$

\section{$3.2 \quad \mathcal{H}$-Representation of Balls}

In this section, we will establish the link between digital disks (discrete polytopes) and $\mathcal{H}$-polytopes.

Definition 3 (Polyhedron). A convex polyhedron is the intersection of a finite set of half-hyperplanes.

Definition 4 (Polytope). A polytope is the convex hull of a finite set of points.

Theorem 1 (Weyl-Minkowski). A subset of Euclidean space is a polytope if and only if it is a bounded convex polyhedron.

As a result, a polytope in $\mathbb{R}^{n}$ can be represented either as the convex hull of its $k$ vertices $(\mathcal{V}$-representation) or by a set of $l$ half-planes ( $\mathcal{H}$-representation):

$$
\begin{gathered}
P=\operatorname{conv}\left(\left\{p_{i}\right\}_{1 \leq i \leq k}\right)=\left\{p=\sum_{i=1}^{k} \alpha_{i} p_{i}: \alpha_{i} \in \mathbb{R}_{+} \text {and } \sum_{i=1}^{k} \alpha_{i}=1\right\}, \\
P=\{x: A x \leq y\},
\end{gathered}
$$

where $A$ is a $l \times n$ matrix, $y$ a vector of $n$ values that we name $\mathcal{H}$-coefficients of $P$. Given two vectors $\boldsymbol{u}$ and $\boldsymbol{v}$, we denote $\boldsymbol{u} \leq \boldsymbol{v}$ if and only if $\forall i, \boldsymbol{u}_{\boldsymbol{i}} \leq \boldsymbol{v}_{\boldsymbol{i}}$. 
Definition 5 (Discrete polytope). A discrete polytope $Q$ is the intersection of a polytope $P$ in $\mathbb{R}$ with $\mathbb{Z}$ (Gauss discretization of $\mathcal{P}$ ).

The minimal parameter representation introduced below is introduced in order to avoid redundancies in the representation.

Definition 6 (Minimal parameter representation). A minimal parameter $\mathcal{H}$-representation of a discrete polytope $Q$, denoted $\widehat{\mathcal{H}}$-representation, is a $\mathcal{H}$ representation of $P=\{x: A x \leq y\}$ such that $y$ is minimal:

$$
P=\left\{x \in \mathbb{Z}^{n}: A x \leq y\right\} \text { and } \forall i \in[1 . . l], \exists x \in P: A_{i} x=y_{i},
$$

where $A_{i}$ stands for the $i^{\text {th }}$ line of the matrix $A$.

The $\widehat{\mathcal{H}}$ function, introduced for convenience, gives the minimal parameter vector for a given polytope $P: \widehat{\mathcal{H}}(P)=\max \{A x: \quad x \in P\}$. As a consequence, $\{x: A x \leq \widehat{\mathcal{H}}(P)\}$ is the $\widehat{\mathcal{H}}$-representation of $P=\{x: A x \leq y\}$.

Definition 7 (Convex discrete set). A Set in $\mathbb{Z}$ is convex if it is a discrete polytope.

By the construction above, any convex discrete set is given by a ( $\widehat{\mathcal{H}}$-representation of a) discrete polytope.

Our main result, Theorem 2 below, gives a link between digital disks and intersection of half-planes in $\mathbb{R}^{2}$. The half-planes are given by the Lambek-Moser inverse of the sequences $f_{i}$ and the matrix $A$. This result will be used as an efficient representation for distance computation and parameter optimization.

Theorem 2. The following statements are equivalent:

$$
\begin{aligned}
D(O, r) & =\left\{p: A_{i} p \leq f_{i}^{\dagger}(r+1), \forall i\right\} \\
d(O, p) & =\max _{i}\left\{f_{i}\left(A_{i} p\right)\right\}
\end{aligned}
$$

Where, by convention, $\forall i, \forall r \leq 0, f_{i}(r)=0$

Proof. Assume that (9) holds. By definition of a disk:

$$
\begin{aligned}
D(O, r) & =\{p: d(O, p) \leq r\} \\
& =\left\{p: \max _{i}\left\{f_{i}\left(A_{i} p\right)\right\} \leq r\right\} \\
& =\left\{p: f_{i}\left(A_{i} p\right) \leq r, \forall i\right\} \\
& =\left\{p: f_{i}\left(A_{i} p\right)<r+1, \forall i\right\} \\
& =\left\{p: A_{i} p \leq f_{i}^{\dagger}(r+1), \forall i\right\}
\end{aligned}
$$


Conversely,

$$
\begin{aligned}
d(O, p) & =\min \{r: p \in D(O, r)\} \\
& =\min \left\{r: A_{i} p \leq f_{i}^{\dagger}(r+1), \forall i\right\} \\
& =\max _{i}\left\{\min \left\{r: A_{i} p \leq f_{i}^{\dagger}(r+1)\right\}\right\} \\
& =\max _{i}\left\{\min \left\{r: f_{i}\left(A_{i} p\right)<r+1\right\}\right\} \\
& =\max _{i}\left\{\min \left\{r: r \geq f_{i}\left(A_{i} p\right)\right\}\right\} \\
& =\max _{i}\left\{f_{i}\left(A_{i} p\right)\right\}
\end{aligned}
$$

Each row $A_{i}$ of the matrix $A$ is a vector normal to a facet of the polytope. The sequence $f_{i}$ represents the speed of the polytope growth in direction $A_{i}$ which does not need to be uniform as illustrated in Fig. 1

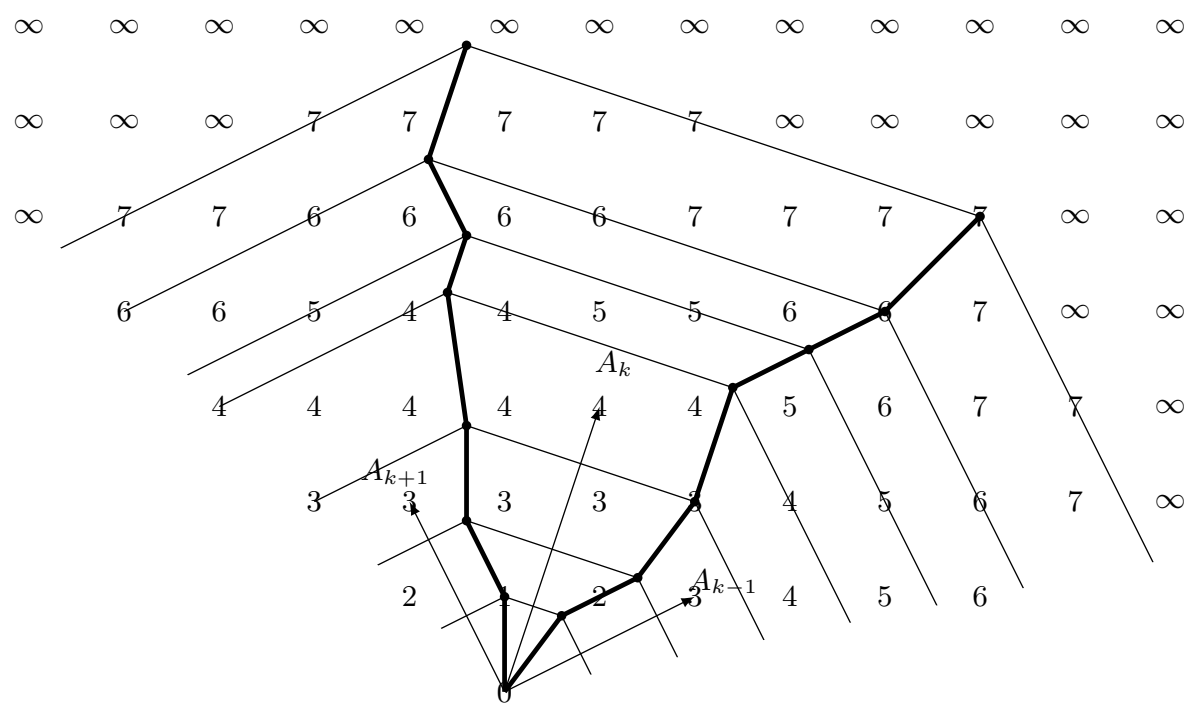

Fig. 1. Illustration of theorem 2] The normal vectors $A_{k-1}$ to $A_{k+1}$ are $(2,1),(1,3)$ and $(-1,2)$. The sequences $f_{k-1}$ to $f_{k+1}$ are respectively $(1,1,2,2,3,3,4,4,5,5,6,6,7,7,7, \bar{\infty}),(1,1,1,2,2,3,3,3,4,4,4,4,5,5,6,6,7,7,7,7, \infty)$ and $(1,1,2,2,3,3,4,4,4,5,6,6,7,7, \bar{\infty})$ and their inverse sequences $f_{k-1}^{\dagger}$ to $f_{k+1}^{\dagger}$ are $(0,2,4,6,8,10,12,15, \overline{16}),(0,3,5,8,12,14,16,20, \overline{24})$ and $(0,2,4,6,9,10,12,14, \overline{15})$. Distance values computed with (9) are given for each discrete point. 


\section{Neighbourhood Sequences and Lambek-Moser Inverse}

\subsection{Weighted Neighbourhood Sequences}

We recall some definitions on weighted neighbourhood sequences from [11. Two grid points $\mathbf{p}_{1}=\left(x_{1}, y_{1}\right), \mathbf{p}_{2}=\left(x_{2}, y_{2}\right) \in \mathbb{Z}^{2}$ are $\rho$-neighbours, $\rho \in\{1,2\}$, if

$$
\begin{aligned}
\left|x_{1}-x_{2}\right|+\left|y_{1}-y_{2}\right| & \leq \rho \text { and } \\
\max \left\{\left|x_{1}-x_{2}\right|,\left|y_{1}-y_{2}\right|\right\} & =1 .
\end{aligned}
$$

The points $\mathbf{p}_{1}, \mathbf{p}_{2}$ are adjacent if $\mathbf{p}_{1}$ and $\mathbf{p}_{2}$ are $\rho$-neighbours for some $\rho$. Two $\rho$ neighbours such that the equality in (10) is attained are called strict $\rho$-neighbours. A ns $B$ is a sequence $B=(b(i))_{i=1}^{\infty}$, where each $b(i)$ denotes a neighbourhood relation in $\mathbb{Z}^{2}$. If $B$ is periodic, i.e., if for some fixed strictly positive $l \in \mathbb{Z}_{+}$, $b(i)=b(i+l)$ is valid for all $i \in \mathbb{Z}_{+}$, then we write $B=(b(1), b(2), \ldots, b(l))$.

We use

$$
\mathbf{1}_{B}(k)=|\{i: b(i)=1,1 \leq i \leq k\}| \text { and } \mathbf{2}_{B}(k)=|\{i: b(i)=2,1 \leq i \leq k\}| .
$$

to denotate the number of 1 :s and 2:s in the ns $B$ up to position $k$.

A path, denoted $\mathcal{P}$, in a grid is a sequence $\mathbf{p}_{0}, \mathbf{p}_{1}, \ldots, \mathbf{p}_{n}$ of adjacent grid points. A path is a $B$-path of length $n$ if, for all $i \in\{1,2, \ldots, n\}, \mathbf{p}_{i-1}$ and $\mathbf{p}_{i}$ are $b(i)$-neighbours. The number of 1 -steps and strict 2 -steps in a given path $\mathcal{P}$ is denoted $\mathbf{1}_{\mathcal{P}}$ and $\mathbf{2}_{\mathcal{P}}$, respectively.

Definition 8. Given the ns $B$, the ns-distance $d\left(\mathbf{p}_{0}, \mathbf{p}_{n} ; B\right)$ between the points $\mathbf{p}_{0}$ and $\mathbf{p}_{n}$ is the length of (one of) the shortest B-path(s) between the points.

Let the real numbers $\alpha$ and $\beta$ (the weights) and a $B$-path $\mathcal{P}$ of length $n$, where exactly $l(l \leq n)$ pairs of adjacent grid points in the path are strict 2-neighbours be given. The cost of the $(\alpha, \beta)$-weighted $B$-path $\mathcal{P}$ is $(n-l) \alpha+l \beta$. The $B$-path $\mathcal{P}$ between the points $\mathbf{p}_{0}$ and $\mathbf{p}_{n}$ is a $(\alpha, \beta)$-weighted minimal cost $B$-path between the points $\mathbf{p}_{0}$ and $\mathbf{p}_{n}$ if no other $(\alpha, \beta)$-weighted $B$-path between the points has lower cost than the $(\alpha, \beta)$-weighted $B$-path $\mathcal{P}$.

Definition 9. Given the ns $B$ and the weights $\alpha, \beta$, the weighted ns-distance $d_{\alpha, \beta}\left(\mathbf{p}_{0}, \mathbf{p}_{n} ; B\right)$ is the cost of (one of) the $(\alpha, \beta)$-weighted minimal cost B-path(s) between the points.

We denote the cumulative sum $B^{\Sigma}(k)$ of the ns $B$ as

$$
B^{\Sigma}(k)=\sum_{l=1}^{k} B(l)=k+\mathbf{2}_{B}(k)
$$

The following construction gives a non-decreasing sequence of integers: $f(k)=$ $B^{\Sigma}(k-1)+1=\mathbf{2}_{B}(k-1)+k$.

Example 1. Given the neighbourhood sequence $B=(1,2,1,2,2)$, the cumulative sum is $B^{\Sigma}(m)=\left\lfloor\frac{8}{5} m\right\rfloor$, its inverse is $B^{\Sigma \dagger}(m)=\left\lceil\frac{5}{8} m-1\right\rceil$, the non-decreasing sequence $f$ defined above is $f(m)=\left\lfloor\frac{8}{5} m-\frac{3}{5}\right\rfloor$, and its inverse is $f^{\dagger}(m)=$ $\left\lceil\frac{5}{8}(m-1)\right\rceil$. 


\begin{tabular}{cccccccccccc}
\hline$m$ & 1 & 2 & 3 & 4 & 5 & 6 & 7 & 8 & 9 & 10 & 11 \\
\hline$B(m)$ & 1 & 2 & 1 & 2 & 2 & 1 & 2 & 1 & 2 & 2 & 1 \\
\hline$B^{\Sigma}(m)$ & 1 & 3 & 4 & 6 & 8 & 9 & 11 & 12 & 14 & 16 & 17 \\
$B^{\Sigma^{\dagger}}(m)$ & 0 & 1 & 1 & 2 & 3 & 3 & 4 & 4 & 5 & 6 & 6 \\
\hline$f(m)$ & 1 & 2 & 4 & 5 & 7 & 9 & 10 & 12 & 13 & 15 & 17 \\
$f^{\dagger}(m)$ & 0 & 1 & 2 & 2 & 3 & 4 & 4 & 5 & 5 & 6 & 7 \\
\hline
\end{tabular}

The following formula for weighted ns-distance is given in [11].

$$
\begin{aligned}
d(0,(x, y)) & =\alpha(2 k-x-y)+\beta(x+y-k), \text { where } \\
k & =\min \left\{l: l \geq x+\max \left\{0, y-\mathbf{2}_{B}(l)\right\}\right\}
\end{aligned}
$$

Proposition 2 below gives an alternative formula for (12). Since the LambekMoser inverse $f^{\dagger}$ of a Beatty sequence can be written on closed form, it is very efficient to compute.

Proposition 2. $\min \left\{l: l \geq x+\max \left\{0, y-\mathbf{2}_{B}(l)\right\}\right\}=\max \left\{x, f^{\dagger}(x+y+1)\right\}$

Proof.

$$
\begin{aligned}
& \left\{k=\min \left\{l: l \geq x+\max \left\{0, y-\mathbf{2}_{B}(l)\right\}\right\}\right\} \\
& \left\{\begin{array}{l}
k \geq \max \left\{x, x+y-\mathbf{2}_{B}(k)\right\} \\
k-1<\max \left\{x, x+y-\mathbf{2}_{B}(k-1)\right\}
\end{array}\right\} \\
& \left\{\begin{array}{l}
k \geq x \\
k \geq x+y-\mathbf{2}_{B}(k) \\
k=x \text { or } k \leq x+y-\mathbf{2}_{B}(k-1)
\end{array}\right\} \\
& \left\{\begin{array}{l}
k \geq x \\
k+\mathbf{2}_{B}(k) \geq x+y \\
k=x \text { or } k+\mathbf{2}_{B}(k-1) \leq x+y
\end{array}\right\} \\
& \left\{\begin{array}{l}
k \geq x \\
f(k+1)-1 \geq x+y \\
k=x \text { or } f(k) \leq x+y
\end{array}\right\} \\
& \left\{\begin{array}{l}
k \geq x \\
k+1>f^{\dagger}(x+y+1) \\
k=x \text { or } k \leq f^{\dagger}(x+y+1)
\end{array}\right\} \\
& \left\{\begin{array}{l}
k \geq x \\
k \geq f^{\dagger}(x+y+1) \\
k=x \text { or } k \leq f^{\dagger}(x+y+1)
\end{array}\right\}
\end{aligned}
$$

Corollary 1. (11) can be rewritten as:

$$
d(0,(x, y))=(2 \alpha-\beta) \max \left\{x, f^{\dagger}(x+y+1)\right\}+(\beta-\alpha)(x+y) .
$$


Example 2. With $\alpha=4, \beta=5$ and $B$ from Example 1

$$
\begin{aligned}
& d(0,(x, y))=3 \max \left\{x, f^{\dagger}(x+y+1)\right\}+x+y \\
& =\max \left\{4 x+y, 3 f^{\dagger}(x+y+1)+x+y\right\} \\
& =\max \left\{f_{1}\left(\left(\begin{array}{ll}
4 & 1
\end{array}\right)\left(\begin{array}{ll}
x & y
\end{array}\right)^{t}\right), f_{2}\left(\left(\begin{array}{ll}
1 & 1
\end{array}\right)\left(\begin{array}{ll}
x & y
\end{array}\right)^{t}\right)\right\}
\end{aligned}
$$

as in (9) where $f_{1}: m \mapsto f_{1}(m)=m$ and $f_{2}: m \mapsto 3 f^{\dagger}(m+1)+m$.

\begin{tabular}{cccccccccccc}
\hline$m$ & 1 & 2 & 3 & 4 & 5 & 6 & 7 & 8 & 9 & 10 & 11 \\
\hline$f_{1}(m)$ & 1 & 2 & 3 & 4 & 5 & 6 & 7 & 8 & 9 & 10 & 11 \\
$f_{1}^{\dagger}(m)$ & 0 & 1 & 2 & 3 & 4 & 5 & 6 & 7 & 8 & 9 & 10 \\
\hline$f_{2}(m)$ & 4 & 8 & 9 & 13 & 17 & 18 & 22 & 23 & 27 & 31 & 32 \\
$f_{2}^{\dagger}(m)$ & 0 & 0 & 0 & 0 & 1 & 1 & 1 & 1 & 2 & 3 & 3 \\
\hline
\end{tabular}

\subsection{Specific Cases}

Example 3 (Special case I-Weighted distances). $B=(2), B^{\Sigma}=(2,4,6, \ldots), f=$ $(1,3,5,7, \ldots)=2 k-1, f^{\dagger}=(0,1,1,2,2,3,3,4,4, \ldots)=\left\lfloor\frac{k}{2}\right\rfloor$ When $x \geq y \geq 0$, $\max \left\{x, f^{\dagger}(x+y+1)\right\}=x$, so (13) becomes:

$$
\begin{aligned}
d(0,(x, y)) & =(2 \alpha-\beta) x+(\beta-\alpha)(x+y) \\
& =\alpha x+(\beta-\alpha) y
\end{aligned}
$$

which is consistent with [2] and can be written in the form of (9) with the matrix $A=\left(\begin{array}{ll}\alpha & \beta-\alpha\end{array}\right)$ and the function $f_{1}: m \mapsto f_{1}(m)=m$ which Lambek-Moser inverse is $f_{1}^{\dagger}: m \mapsto m-1$. A similar distance formulation for chamfer norms with arbitrary large masks was given in [6, (20)].

Example 4 (Special case II - ns-distances). A ns-distance is a special case of wnsdistance for which path costs are computed with unitary weights $(\alpha=\beta=1)$. Then (13) becomes:

$$
d(0,(x, y))=\max \left\{x, f^{\dagger}(x+y+1)\right\}
$$

This can be written in the form of (9) with $A=\left(\begin{array}{cc}\alpha & \beta-\alpha \\ 1 & 1\end{array}\right)=\left(\begin{array}{ll}1 & 0 \\ 1 & 1\end{array}\right)$, $f_{1}: m \mapsto m$ and $f_{2}: m \mapsto f^{\dagger}(m+1)$. The Lambek-Moser inverses of $f_{1}$ and $f_{2}$ are $f_{1}^{\dagger}: m \mapsto m-1$ and $f_{2}^{\dagger}: m \mapsto f(m)-1$. I.e.

$$
\begin{aligned}
D(0, r) & =\left\{p: g_{i}\left(A_{i} p\right)<r+1, \forall i\right\} \\
& =\left\{p: A_{i} p \leq f_{i}^{\dagger}(r+1), \forall i\right\} \\
& =\left\{p:\left(\begin{array}{ll}
1 & 0 \\
1 & 1
\end{array}\right) p \leq\left(\begin{array}{l}
f_{1}^{\dagger}(r+1) \\
f_{2}^{\dagger}(r+1)
\end{array}\right)\right\}
\end{aligned}
$$




\begin{tabular}{cccccccccccc}
\hline$m$ & 1 & 2 & 3 & 4 & 5 & 6 & 7 & 8 & 9 & 10 & 11 \\
\hline$B(m)$ & 1 & 2 & 1 & 2 & 2 & 1 & 2 & 1 & 2 & 2 & 1 \\
\hline$f^{\dagger}(m)$ & 0 & 1 & 2 & 2 & 3 & 4 & 4 & 5 & 5 & 6 & 7 \\
\hline$f_{1}(m)$ & 1 & 2 & 3 & 4 & 5 & 6 & 7 & 8 & 9 & 10 & 11 \\
$f_{1}^{\dagger}(m)$ & 0 & 1 & 2 & 3 & 4 & 5 & 6 & 7 & 8 & 9 & 10 \\
\hline$f_{2}(m)$ & 1 & 2 & 2 & 3 & 4 & 4 & 5 & 5 & 6 & 7 & 7 \\
$f_{2}^{\dagger}(m)$ & 0 & 1 & 3 & 4 & 6 & 8 & 9 & 11 & 12 & 14 & 16 \\
\hline
\end{tabular}

\section{Optimization}

In this section, we will find parameters $\alpha, \beta, \tau$ that minimize the rotational dependency of the wns-distance. The digital disk obtained by wns is

$$
D(0, r)=\left\{p: A_{i} p \leq f_{i}^{\dagger}(r+1), \forall i\right\}
$$

Now, we restrict the cumulative neighbourhood sequence $B^{\Sigma}$ to rational Beatty sequences, i.e. on the form $B^{\Sigma}(m)=\lfloor\tau m\rfloor, 1 \leq \tau \leq 2$.

$$
\begin{array}{r}
\alpha x_{1}+(\beta-\alpha) y_{1} \leq f_{1}^{\dagger}(r+1) \\
x_{2}+y_{2} \leq f_{2}^{\dagger}(r+1)
\end{array}
$$

With $f_{1}^{\dagger}(r+1)=r$ and equality above and $x=x_{1}=x_{2}$ and $y=y_{1}=y_{2}$, we calculate the coordinates of the vertices of the $\mathcal{H}$-polytopes:

$$
\begin{aligned}
& x=\frac{r+(\alpha-\beta) f_{2}^{\dagger}(r+1)}{2 \alpha-\beta} \\
& y=\frac{\alpha f_{2}^{\dagger}(r+1)-r}{2 \alpha-\beta}
\end{aligned}
$$

With equality above and $x=x_{1}$ and $y_{2}=y_{1}=0$,

$$
x=\frac{r}{\alpha}
$$

By symmetry, the whole polygon is given by the vertices

$$
\left(\frac{r+(\alpha-\beta) f_{2}^{\dagger}(r+1)}{2 \alpha-\beta}, \frac{\alpha f_{2}^{\dagger}(r+1)-r}{2 \alpha-\beta}\right),\left(\frac{r}{\alpha}, 0\right) .
$$

Optimization Procedure. Loop over integer $\alpha, \beta$ in some predefined interval and rational $\tau$ (that defines the Beatty sequence) between 1 and 2 .

Find the parameters $\alpha, \beta, \tau$ that gives the minimum $P^{2} A$ for each value up to $R_{\max }$ that is attained for the specific parameters. 
Example 5. $\tau$ : 500 uniform samples between 1 and $2.1 \leq \alpha \leq \beta \leq 10$ (and $2 \alpha \leq \beta)$.

\begin{tabular}{ccccc}
\hline radius & $\tau$ & $\alpha$ & $\beta$ & mean $P^{2} A /(4 \pi)$ \\
\hline$\alpha$ & 2 & $t$ & $t$ & 1.27 \\
\hline $2 \alpha$ & 1.5 & $t$ & $t$ & 1.1667 \\
\hline $3 \alpha$ & 2 & 3 & 4 & 1.1207 \\
\hline $4 \alpha$ & 2 & 7 & 9 & 1.0937 \\
\hline $5 \alpha$ & 2 & 7 & 9 & 1.0748 \\
\hline $6 \alpha$ & 2 & 7 & 9 & 1.0658 \\
\hline $7 \alpha$ & 2 & 7 & 9 & 1.0605 \\
\hline $8 \alpha$ & 2 & 7 & 9 & 1.0567 \\
\hline $9 \alpha$ & 1.834 & 7 & 9 & 1.0537 \\
\hline $10 \alpha$ & 1.834 & 7 & 9 & 1.0489 \\
\hline
\end{tabular}

Note that in Example 5. the neighbourhood sequence always start with a 1 due to the definition of $f$. In Example [6] we use $f^{\prime}(k)=f(k)+1$ instead, which means that the first element in $B$ instead is always a 2.

Example 6. $\tau$ : 500 uniform samples between 1 and $2.1 \leq \alpha \leq \beta \leq 10$ (and $2 \alpha \leq \beta) \cdot f^{\prime}(k)=f(k)+1$

\begin{tabular}{ccccc}
\hline radius & $\tau$ & $\alpha$ & $\beta$ & mean $P^{2} A /(4 \pi)$ \\
\hline$\alpha$ & 1 & $t$ & $t$ & 1.1312 \\
\hline $2 \alpha$ & 1 & $t$ & $t$ & 1.1312 \\
\hline $3 \alpha$ & 2 & 5 & 6 & 1.1081 \\
\hline $4 \alpha$ & 1.6680 & 4 & 5 & 1.1024 \\
\hline $5 \alpha$ & 1.75 & 7 & 9 & 1.0758 \\
\hline $6 \alpha$ & 1.75 & 7 & 9 & 1.0644 \\
\hline $7 \alpha$ & 1.75 & 7 & 9 & 1.0567 \\
\hline $8 \alpha$ & 1.6680 & 7 & 9 & 1.0504 \\
\hline $9 \alpha$ & 1.6680 & 7 & 9 & 1.0458 \\
\hline $10 \alpha$ & 1.6680 & 7 & 9 & 1.0424 \\
\hline
\end{tabular}




\section{Conclusions}

In this paper, we express digital distance functions in terms of integer sequences. Our main result, Theorem 2, gives a link between digital distance functions that can be written on the form (9) and the corresponding digital disks. The obtained expressions are elegant and, most importantly, can be computed efficiently. Since the inverse of Beatty sequences can be computed in constant time, this holds also for distance functions written on the form given in Theorem 2

We give examples on how the new way of expressing the distance functions can be applied to well-known digital distance functions such as weighted distances and distances based on neighbourhood sequences. The so-obtained formulas are used to find optimal parameters for short neighbourhood sequences.

We also believe that the results presented in this paper has the potential of having large impact on the development of the theory on digital distance functions.

\section{References}

1. Beatty, S.: Problem 3173. The American Mathematical Monthly 33(3), 159 (1926)

2. Borgefors, G.: Distance transformations in arbitrary dimensions. Computer Vision, Graphics, and Image Processing 27(3), 321-345 (1984)

3. Hajdu, A., Hajdu, L.: Approximating the Euclidean distance using non-periodic neighbourhood sequences. Discrete Mathematics 283(1-3), 101-111 (2004)

4. Lambek, J., Moser, L.: Inverse and complementary sequences of natural numbers. The American Mathematical Monthly 61(7), 454-458 (1954)

5. Montanari, U.: A method for obtaining skeletons using a quasi-Euclidean distance. Journal of the ACM 15(4), 600-624 (1968)

6. Normand, N., Evenou, P.: Medial axis lookup table and test neighborhood computation for 3D chamfer norms. Pattern Recognition 42(10), 2288-2296 (2009)

7. Normand, N., Strand, R., Evenou, P., Arlicot, A.: Path-Based Distance with Varying Weights and Neighborhood Sequences. In: Debled-Rennesson, I., Domenjoud, E., Kerautret, B., Even, P. (eds.) DGCI 2011. LNCS, vol. 6607, pp. 199-210. Springer, Heidelberg (2011)

8. Normand, N., Strand, R., Evenou, P., Arlicot, A.: Minimal-delay distance transform for neighborhood-sequence distances in 2D and 3D. Computer Vision and Image Understanding (2013)

9. Rosenfeld, A., Pfaltz, J.L.: Sequential operations in digital picture processing. Journal of the ACM 13(4), 471-494 (1966)

10. Rosenfeld, A., Pfaltz, J.L.: Distances functions on digital pictures. Pattern Recognition 1(1), 33-61 (1968)

11. Strand, R.: Weighted distances based on neighbourhood sequences. Pattern Recognition Letters 28(15), 2029-2036 (2007)

12. Verwer, B.J.H., Verbeek, P.W., Dekker, S.T.: An efficient uniform cost algorithm applied to distance transforms. IEEE Transactions on Pattern Analysis and Machine Intelligence 11(4), 425-429 (1989) 